\title{
DIMENSIONES ÉTICAS DE LA CRÍTICA AGROECOLÓGICA A LA BIOTECNOLOGÍA AGRÍCOLA
}

\author{
Miguel A. Altieri*
}

\begin{abstract}
Resumen: El objetivo del presente trabajo es cuestionar las falsas promesas hechas por la industria de la ingeniería genética agrícola, al prometer que los cultivos producidos mediante esta tecnología generarían una agricultura menos dependendiente en insumos químicos, aumentarían la productividad y ayudarían a reducir los problemas ambientales. Este artículo también analiza las críticas ambientalistas a la biotecnología, expresadas en la preocupación por sus efectos sobre las condiciones sociales y económicas, y los valores culturales, religiosos y morales de las diferentes culturas, que han sido ignorados reiteradamente en el desarrollo tecnológico de los OvGM. El autor concluye afirmando que ha llegado el momento de enfrentar socialmente el reto y la realidad de la ingeniería genética. Las compañías de biotecnología deben sentir el impacto de los movimientos ambientalistas, laborales y campesinos, de modo que reorienten su trabajo para el beneficio de toda la sociedad y de la naturaleza.
\end{abstract}

Palabras clave: Agrobiotecnología, OGM, agroecología, biotecnología industrial

\section{ETHICAL DIMENSIONS OF AGROECOLOGY CRITICISM TO THE AGROBIOTECHNOLOGY}

\begin{abstract}
The goal of this report is to put into question the false promises that agroindustry has made about genetic engineering in agriculture. They have promised that the transgenic crops should generate agriculture less dependent of chemical supplies, it should increase the productivity and it would help to reduce the environmental problems. On the other hand, this paper analyzes the environmentalist criticism to the biotechnology, specially the deep worry about the social, economic moral, religious, and cultural effects or values that have been ignored in the biotechnological development of the agrobiotechnology. The author concludes starting that it has arrived the social moment to challenge the reality of genetic engineering. The biotechnology companies must feel the impact of environmentalist, labor and rural movements, so that they may reorient their work for the benefit of society and nature.
\end{abstract}

Key words: Agrobiotechnology, GMO, agroecology, industrial biotechnology

\section{DIMENSÕES ÉTICAS DA CRÍTICA AGRO ECOLÓGICA À BIOTECNOLOGIA AGRÍCOLA}

Resumo: O objetivo do presente trabalho é questionar as falsas promessas feitos pela indústria da engenharia genética agrícola, ao prometer que os cultivos produzidos mediante esta tecnologia gerariam uma agricultura menos dependente de insumos químicos, aumentariam a produtividade e ajudariam a reduzir os problemas ambientais. Este artigo também analisa as críticas ambientalistas à biotecnologia, expressas na preocupação por seus efeitos sobre as condições sociais e econômicas, e os valores culturais, religiosos e morais das diferentes culturas, que foram ignorados sistematicamente no desenvolvimento tecnológico dos OGMs. O autor conclui afirmando que chegou o momento de enfrentar socialmente o desafio e a realidade da engenharia genética. As empresas de biotecnologia devem sentir o impacto dos movimentos ambientalistas, laborais e rurais, de modo que reorietem seu trabalho para o benefício de toda a sociedade e da natureza.

Palavras chave: Agro biotecnologia, OGMs, agro ecologia, biotecnologia industrial

* Professor of Agroecology, Division of Insect Biology, University of California.

Correspondencia: agroeco3@nature.berkeley.edu 


\section{Introducción}

El problema del hambre y la pobreza en el mundo, cuyas raíces se asientan en la desigual distribución de los recursos productivos y en la injusticia social que prevalecen principalmente en los países del Sur, pretendió ser enfrentado hace cincuenta años mediante las innovaciones tecnológicas de la Revolución Verde. Sin embargo, aun cuando se incrementaron los rendimientos de determinados cultivos, éstos se concentraron en productos de exportación y agroindustriales bajo sistemas de monoproducción a gran escala, sin un impacto significativo en la pequeña agricultura y, por ende, en la pobreza rural.

A inicios del tercer milenio, el hambre y la pobreza mundial se han incrementado, a pesar de que los excedentes alimenticios acumulados en los países desarrollados servirían para enfrentar decididamente este problema. Sin tomar en cuenta esta realidad, los grupos económicos que estuvieron detrás de la Revolución Verde (empresas de semillas y agroquímicos) argumentan, una vez más, que el problema está en el incremento de la productividad agrícola y promueven una Segunda Revolución Verde, basada en la biotecnología y en la ingeniería genética. Las corporaciones de agroquímicos, las cuales controlan cada vez más la orientación y las metas de la innovación agrícola, sostienen que la ingeniería genética mejorará la sostenibilidad de la agricultura al resolver los muchos problemas que afectan a la agricultura industrial y librará al Tercer Mundo de la baja productividad, la pobreza y el hambre.

Comparando mito y realidad, el objetivo de este artículo es cuestionar las falsas promesas hechas por la industria de la ingeniería genética. Ellos han prometido que los cultivos producidos por ingeniería genética harán la agricultura menos dependendiente en insumos químicos, aumentarán la productividad, disminuirán los costos de insumos y ayudarán a reducir los problemas ambientales. Al cuestionar los mitos de la biotecnología, aquí se muestra a la ingeniería genética como lo que realmente es: otra ronda tecnológica o "varita mágica" destinada a entrampar los problemas ambientales de la agricultura sin cuestionar las raíces causales que ocasionaron los problemas la primera vez(1). La biotecnología desarrolla soluciones monogénicas, diseñadas sobre modelos industriales de eficiencia, para problemas que derivan de sistemas de monocultivo ecológicamente inestables. Se ha probado ya que, en el caso de los plaguicidas, tal enfoque unilateral no fue ecológicamente confiable. Este enfoque, unilateral y reduccionista, que utilizó el paradigma "un químico-una plaga" y que llevó a problemas de resistencias de plagas y contaminación ambiental, no difiere del enfoque biotecnológico que ahora enfatiza un paradigma comparablemente reduccionista de "un genuna plaga" y el cual conlleva a las mismas falacias ecológicas.

La agricultura industrial moderna, hoy caracterizada por el modelo de la biotecnología, se basa en una premisa filosófica que es fundamentalmente errónea y que necesita ser expuesta y criticada para avanzar hacia una agricultura verdaderamente sostenible. Esto es particularmente relevante en el caso de la biotecnología, donde la alianza de la ciencia reduccionista y la industria multinacional monopolizadora lleva a la agricultura por un camino equivocado. La biotecnología percibe los problemas agrícolas como deficiencias genéticas de los organismos y trata a la naturaleza como una mercancía y, en el camino, hace a los agricultores más dependientes de un sector de agronegocios que concentra cada vez más su poder sobre el sistema alimentario. Esto es preocupante, especialmente hoy en que son las motivaciones económicas, más que las preocupaciones sobre el medio ambiente, las que determinan el tipo de investigación y las modalida- 
des de producción agrícola que prevalecen en todo el mundo(2).

\section{Cuestionamiento Ético de la Biotecnología}

Las críticas ambientalistas a la biotecnología cuestionan las suposiciones de que la ciencia de la biotecnología está libre de valores y que no puede estar equivocada o ser mal utilizada, y piden una evaluación ética de la investigación en ingeniería genética y sus productos(3). Se considera que quienes promueven la biotecnología tienen una visión utilitaria de la naturaleza y favorecen el libre intercambio (trade-off) de las ganancias económicas por el daño ecológico, manteniéndose indiferentes ante las consecuencias que acarrea para los seres humanos. En el corazón de la crítica están los efectos biotecnológicos sobre las condiciones sociales y económicas y los valores culturales, religiosos y morales que se han ignorado. Todo esto ha llevado a una serie de científicos y filósofos a plantearse una serie de preguntas que abordan temas tales como:

Los propósitos de la tecnología: ¿Cuál es la real necesidad de esta tecnología? ¿A qué necesidades responde la biotecnología? ¿Quién dice qué se necesita y por qué? ¿Qué problemas va a solucionar? ¿Cuál es la causa de estos problemas y acaso la biotecnología realmente los enfrenta de raíz? ¿Cómo afecta la tecnología a lo que se está produciendo? ¿Cómo, para qué y para quién se está produciendo? ¿Cuáles son las metas sociales y los criterios éticos que guían la investigación biotecnológica?

Definiciones de riesgo: ¿Se han considerado los efectos ambientales, sociales, económicos y políticos? ¿Quién ha definido el alcance y significancia de estos efectos? ¿Son los efectos irreversibles? ¿Se están midiendo estos efectos en comparación con tecnologías más blandas existentes? ¿Cómo se determina la aceptabilidad del riesgo y quién lo hace? ¿Acaso la biotecnología cierra opciones para el futuro? ¿O se promueve a expensas de otros enfoques? ¿Cuáles han sido las alternativas ignoradas? ¿Cómo se estiman los beneficios?

Distribución de riesgos y beneficios: ¿Cómo se distribuyen los costos y beneficios? ¿Quién se beneficia de la biotecnología? ¿Quién pierde? ¿Cómo se determina y por quién que la distribución es aceptable? ¿Acaso la biotecnología concentra poder y en manos de quién?

Alternativas: ¿Se han explorado a fondo alternativas para solucionar problemas que la biotecnología dice solucionar? ¿Esta exploración examina una serie de intereses e incluye la participación de todos los grupos sociales? ¿Está el proceso abierto al escrutinio público? ¿Se distribuyen equitativamente los fondos de investigacion para las alternativas? ¿Existen alternativas ambientalmente menos nocivas $\mathrm{y}$ socialmente más justas?

\section{Biotecnología y Hambre}

Las compañías de biotecnología sostienen que los organismos genéticamente modificados (GMOs en inglés) -específicamente las semillas genéticamente alteradas- son hallazgos científicos necesarios para alimentar al mundo y reducir la pobreza en los países en desarrollo. La mayoría de las organizaciones internacionales encargadas de la política y de la investigación para el mejoramiento de la seguridad alimentaria en el mundo en desarro1lo hacen eco de este punto de vista. Este punto se basa en dos suposiciones críticas: que el hambre se debe a una brecha entre la producción de alimentos y la densidad de la población humana o la tasa de crecimiento; y que la ingeniería genética es la única o la mejor forma de incrementar la producción agrícola y por lo tanto de cubrir las futuras necesidades de alimento. 
Un punto inicial para aclarar estas falsas concepciones es entender que no hay una relación entre la presencia del hambre en un país determinado y su población. Por cada nación hambrienta y densamente poblada como Bangladesh o Haití, hay un país escasamente poblado y hambriento como Brasil o Indonesia. El mundo produce hoy más alimentos por habitante que nunca antes. Hay suficiente alimento disponible para proveer 4,3 libras por persona cada día; 2,5 libras de granos, frijoles y nueces; alrededor de una libra de carne, leche y huevos y otra de frutas y verduras(4).

En 1999 se produjo suficiente cantidad de granos en el mundo para alimentar una población de ocho mil millones de personas (seis mil millones habitaron el planeta en el 2000), si éstos se distribuyeran equitativamente o no se dieran como alimento a los animales. Siete de cada diez libras de granos se usan para alimentar animales en Estados Unidos. Países como Brasil, Paraguay, Tailandia e Indonesia dedican miles de acres de tierras agrícolas a la producción de soya y yuca para exportar a Europa como alimento para el ganado. Canalizando un tercio de los granos producidos en el mundo hacia la población hambrienta y necesitada, el hambre cesaría instantáneamente(4).

El hambre también ha sido creada por la globalización, especialmente cuando los países en desarrollo adoptan las políticas de libre comercio recomendadas por agencias internacionales (reduciendo los aranceles y permitiendo el flujo de los productos de los países industrializados). La experiencia de Haití, uno de los países más pobres del mundo, es ilustrativa. En 1986 Haití importó sólo 7000 toneladas de arroz, porque la mayor parte se producía en la isla. Cuando abrió su economía al mundo, los inundó un arroz más barato proveniente de los Estados Unidos, donde la industria del arroz es subsidiada. En 1996, Haití importó 196000 toneladas de arroz foráneo al costo de US\$ 100 millones anuales. La producción de arroz haitiano se volvió insignificante cuando se concretó la dependencia en el arroz extranjero. El hambre se incrementó(5).

Las causas reales del hambre son la pobreza, la desigualdad y la falta de acceso a los alimentos y a la tierra. Demasiada gente es muy pobre (alrededor de dos mil millones sobreviven con menos de un dólar al día) para comprar los alimentos disponibles (a menudo con una pobre distribución) o carecen de tierra y los recursos para sembrarla(4). Porque la verdadera raíz del hambre es la desigualdad, cualquier método diseñado para reforzar la producción de alimentos, pero que agudice esta desigualdad, fracasará en reducir el hambre. Por el contrario, sólo las tecnologías que tengan efectos positivos en la distribución de la riqueza, el ingreso y los activos, que estén a favor de los pobres, podrán en realidad reducir el hambre. Afortunadamente, tales tecnologías existen y pueden agruparse bajo la disciplina de la agroecología $(6,7)$.

Atacando la desigualdad por medio de reformas agrarias se mantiene la promesa de un aumento de la productividad que sobrepasa el potencial de la biotecnología agrícola. Mientras que los defensores de la industria hacen una promesa de 15, 20 e incluso $30 \%$ de aumento de los rendimientos por la biotecnología, los pequeños agricultores producen hoy de 200 a $1000 \%$ más por unidad de área que las grandes fincas a nivel mundial(8). Una estrategia clara para tomar ventaja de la productividad de las pequeñas fincas es impulsar reformas agrarias que reduzcan las grandes propiedades ineficientes e improductivas a un tamaño pequeño óptimo y, así, proporcionar las bases para el incremento de la producción en fincas de pequeños agricultores, incrementos ante los cuales se diluiría la publicitada promesa productiva de la biotecnología. 
Es importante entender que la mayor parte de innovaciones en la biotecnología agrícola se orientan a las ganancias más que a las necesidades. El verdadero motor de la industria de la ingeniería genética no es hacer la agricultura más productiva, sino generar mayores ingre$\operatorname{sos}(2)$. Esto se ilustra revisando las principales tecnologías del mercado de hoy: (i) cultivos resistentes a los herbicidas, tales como la Soya Ready Roundup de Monsanto, semillas que son tolerantes al herbicida Roundup de Monsanto, y (ii) los cultivos Bt (Bacillus thuringiensis) que han sido desarrollados por ingeniería genética para producir su propio insecticida. En el primer caso, la meta es ganar más participación del mercado de los herbicidas para un producto exclusivo y, en el segundo, aumentar las ventas de semillas aun a costa de dañar la utilidad de un producto clave para el manejo de plagas (el insecticida microbiano a base de Bt) en el que confían muchos agricultores -incluyendo a la mayoría de agricultores de cultivos orgánicos- como una poderosa alternativa a los insecticidas.

Estas tecnologías responden a la necesidad de las compañías de biotecnología de intensificar la dependencia de los agricultores de semillas protegidas por la llamada "propiedad intelectual", lo que entra en conflicto directamente con los antiguos derechos de los agricultores a reproducir, compartir o almacenar semillas(9). Cada vez que pueden, las corporaciones obligan a los agricultores a comprar una marca de insumos de la compañía y les prohíben guardar o vender la semilla. Si los agricultores de los Estados Unidos adoptan soya transgénica, deben firmar un acuerdo con Monsanto. Si siembran soya transgénica al año siguiente, la multa es de unos US\$ 3000 por acre, dependiendo del área. Esta multa puede costarle al agricultor su finca, es decir, su hogar. Controlando el germoplasma, desde la producción de semillas hasta su venta, y obligando a los agricultores a pagar precios inflados por paquetes de semillas-químicos, las compañías están decididas a extraer el máximo beneficio de su inversión(3).

\section{La Biotecnología responde: El Arroz Dorado, solución ciega a la ceguera}

Los científicos que apoyan la biotecnología y están en desacuerdo con la afirmación de que la mayor parte de la investigación en biotecnología está basada en el lucro más que en la necesidad, usan como parte de su retórica humanitaria el recientemente desarrollado arroz dorado, todavía no comercializado. Este arroz experimental es rico en betacaroteno, el precursor de la vitamina $A$, que es un producto nutritivo importante para millones de niños, especialmente en Asia, quienes sufren de deficiencia de Vitamina A, lo que puede conducir a la ceguera.

Quienes han creado el arroz dorado dicen que este nuevo cultivo fue desarrollado con fondos públicos y que una vez que se demuestre su viabilidad en campos de cultivo, será distribuido gratuitamente entre los pobres. La idea de que un arroz genéticamente alterado es la forma apropiada de tratar la condición de dos millones de niños en riesgo de ceguera -inducida por deficiencia de Vitamina A-, revela una tremenda ingenuidad sobre las causas reales de la malnutrición por falta de vitaminas y micronutrientes. Si nos remitimos a los patrones de desarrollo y nutrición humanos, rápidamente nos damos cuenta de que la deficiencia de Vitamina A no está caracterizada como un problema sino como un síntoma, una señal de alerta. Nos alerta sobre mayores deficiencias asociadas tanto con la pobreza como con el cambio en la agricultura (desde sistemas de cultivo diversificados hacia monocultivos) promovido por la Revolución Verde.

La gente no presenta deficiencia de Vitamina A porque el arroz contiene muy poca Vita- 
mina A, o betacaroteno, sino porque su dieta se reduce solamente a arroz y a casi nada más, y sufren de otras enfermedades nutricionales que no se pueden tratar con betacaroteno, pero que podrían ser tratadas, junto con la deficiencia de Vitamina A, con una dieta más variada. El arroz dorado debe ser considerado un intento unidimensional de reparar un problema creado por la Revolución Verde: el problema de la disminución de la variedad de cultivos y la diversidad en la dieta.

Una solución de "varita mágica" que coloca betacaroteno en el arroz -con potencial daño ecológico y a la salud-, al tiempo que deja intacta a la pobreza, las dietas pobres y el monocultivo extensivo, no puede hacer ninguna contribución duradera al bienestar. Usando la frase de Vandana Shiva, "un enfoque de esa naturaleza revela ceguera ante las soluciones sencillas disponibles para combatir la ceguera inducida por la deficiencia de Vitamina A, que incluye a muchas plantas que, cuando son introducidas (o reintroducidas) en la dieta, proporcionan el beta caroteno y otras vitaminas y micronutrientes".

Aunque los vegetales silvestres han sido considerados periféricos en los hogares campesinos, su recolección, tal como se practica actualmente en muchas comunidades rurales, constituye un aditivo significativo a la nutrición y subsistencia de las familias campesinas. Dentro y fuera de la periferia de las pozas de arroz hay abundantes vegetales de hoja verde, silvestres y cultivados, ricos en vitaminas y nutrientes, muchos de los cuales son eliminados cuando los agricultores adoptan el monocultivo y los herbicidas asociados(10).

Los biotecnólogos del arroz no entienden las profundas tradiciones culturales populares que determinan las preferencias de alimentos entre la población asiática, especialmente el significado social e incluso religioso del arroz blanco. Es altamente improbable que el arroz dorado reemplace al arroz blanco que por milenios ha jugado variados papeles en aspectos nutricionales, culinarios y ceremoniales. No cabe duda de que el arroz dorado sacudirá las tradiciones asociadas con el arroz blanco en la misma forma en que lo harían las papas fritas verdes o azules en las preferencias de la gente de los Estados Unidos.

Pero incluso si el arroz dorado ingresa en los platos de los pobres de Asia, no hay una garantía de que ello beneficiará a la gente pobre que no come alimentos ricos en grasas o aceites. El betacaroteno es soluble en grasas y su ingestión por el intestino depende de la grasa o aceite de la dieta. Aún más, las personas que sufren de desnutrición proteica y carecen de dietas ricas en grasas no pueden almacenar bien la Vitamina A en el hígado ni pueden transportarla a los diferentes tejidos corporales donde se requiere. Debido a la baja concentración de betacaroteno en el arroz milagroso (aproximadamente 1,5 microgramos por gramo de peso fresco) habría que comer más de un kilogramo de arroz diario para obtener la ración diaria recomendada de Vitamina A. Cualquier planta silvestre comestible y que abundaba en los arrozales antes de que los herbicidas de la revolucion verde los eliminara, contiene sobre 100 microgramos de betacaroteno.

\section{Los Mitos de la Biotecnología}

Mito 1: La biotecnología beneficiará a los agricultores de Estados Unidos y del mundo desarrollado.

La mayoría de las innovaciones en biotecnología agrícola son motivadas más por criterios económicos que por necesidades humanas. Por lo tanto, la finalidad de la industria de la ingeniería genética no es resolver problemas agrícolas, sino obtener ganancias. Más aún, la biotecnología busca industrializar en mayor 
grado la agricultura e intensificar la dependencia de los agricultores de los insumos industriales, ayudados por un sistema de derechos de propiedad intelectual que legalmente inhibe los derechos de los agricultores a reproducir, intercambiar y almacenar semillas(2). Al controlar el germoplasma desde la semilla hasta la venta y forzar a los agricultores a pagar precios inflados por los paquetes de semilla-química, las compañías están dispuestas a obtener el mayor provecho de su inversión.

Debido a que las biotecnologías requieren grandes capitales, continuarán condicionando el patrón de cambio de la agricultura en los Estados Unidos, aumentando la concentración de la producción agrícola en manos de las grandes corporaciones. Como en el caso de otras tecnologías que ahorran mano de obra al aumentar la productividad, la biotecnología tiende a reducir los precios de los bienes y a poner en marcha una maquinaria tecnológica que deja fuera del negocio a un número significativo de agricultores. En Estados Unidos quiebran alrededor de 211 agricultores por día. El ejemplo de la hormona de crecimiento bovino, confirma la hipótesis de que la biotecnología acelerará la desaparición de las pequeñas fincas lecheras(3). En Argentina, la comercializacion de la soya transgénica resistente al Roundup abrió las puertas para la expansión de la labranza mínima de 7 mil hectáreas en 1992 a un millón en el año 2000, cuando el uso de Roundup alcanzó los $100 \mathrm{mi}-$ llones de litros. Pero lo trágico fue que, en la medida que se expandía el área bajo soya transgénica, el número de agricultores decreció de 170 a 116 mil, mientras el tamaño de la finca se incrementó. Como la soya transgénica facilita el manejo de malezas, permite a los agricultores con más capital expandir su área pues, en economías neoliberales, la única fórmula de sobrevivir es "hacerse grande o fracasar" (get big or get out). Claramente la biotecnología en este caso sirve de instrumento de concentración de tierras.
Mito 2: La biotecnología beneficiará a los pequeños agricultores y favorecerá a los hambrientos y pobres del tercer mundo.

Si la Revolución Verde ignoró a los pequeños agricultores y a los de escasos recursos, la biotecnología exacerbará aún más la marginación porque tales tecnologías, que están bajo el control de corporaciones y protegidas por patentes, son costosas e inapropiadas para las necesidades y circunstancias de los grupos indígenas y campesinos. Ya que la biotecnología es una actividad principalmente comercial, esta realidad determina las prioridades de qué investigar, cómo se aplica y a quién beneficiará. Mientras el mundo carece de alimentos y sufre de contaminación por plaguicidas, el foco de las corporaciones multinacionales es la ganancia, no la filantropía. Esta es la razón por la cual los biotecnólogos diseñan cultivos transgénicos para nuevos tipos de mercado o para sustitución de las importaciones, en lugar de buscar mayor producción de alimentos. En general, las compañías de biotecnología ponen énfasis en un rango limitado de cultivos para los cuales hay mercados grandes y seguros, dirigidos a sistemas de producción de grandes capitales. Como los cultivos transgénicos son plantas patentadas, esto significa que los campesinos pueden perder sus derechos sobre su propio germoplasma regional y no se les permitirá, según la Organización Mundial de Comercio (OMC), reproducir, intercambiar o almacenar semillas de su cosecha. Es difícil concebir cómo se introducirá este tipo de tecnología en los países del tercer mundo de modo que favorezca a las masas de agricultores pobres.

Si los biotecnólogos estuvieran realmente comprometidos en alimentar al mundo, ¿por qué los genios de la biotecnología no se vuelcan a desarrollar nuevas variedades de cultivos más tolerantes a las malezas en vez de a los 
herbicidas? ¿O por qué no se desarrollan productos de biotecnología más promisorios, como plantas fijadoras de nitrógeno o tolerantes a la sequía? Los productos de la biotecnología debilitarán las exportaciones de los países del tercer mundo, especialmente de los productores de pequeña escala. El desarrollo, por medio de la biotecnología, del producto "Thaumatin" es apenas el comienzo de una transición hacia edulcorantes alternativos que, en el futuro, reemplazarán al mercado del azúcar del tercer mundo. Se estima que alrededor de 10 millones de agricultores de caña de azúcar en el tercer mundo podrían enfrentar la pérdida de su sustento cuando los edulcorantes procesados en laboratorio comiencen a invadir los mercados mundiales. La fructosa producida por la biotecnología ha capturado ya cerca del $10 \%$ del mercado mundial y ha causado la caída de los precios del azúcar, dejando desempleados a cientos de miles de trabajadores. Pero tal limitación de las oportunidades rurales no se reduce a los edulcorantes. Unos 70000 agricultores productores de vainilla en Madagascar quedaron en la ruina cuando una firma de Texas produjo vainilla en sus laboratorios de biotecnología(2).

La expansión de las palmas aceiteras clonadas por Unilever incrementará de manera sustancial la producción de aceite de palma con dramáticas consecuencias para los agricultores que producen otros aceites vegetales (de maní en Senegal y de coco en Filipinas).

Mito 3: La biotecnología no atentará contra la soberanía ecológica del tercer mundo.

Desde que el norte se dio cuenta de los servicios ecológicos que proporciona la biodiversidad, de los cuales el sur es el mayor repositorio, el tercer mundo ha sido testigo de una "fiebre genética", en la medida en que las corporaciones multinacionales exploran los bosques, campos de cultivos y costas en busca del oro genético del sur. Protegidas por la OMC, estas corporaciones practican libremente la "biopiratería", la cual cuesta a las naciones en desarrollo, según la Fundación para el Avance Rural (RAFI), unos US\$ 4500 millones al año por la pérdida de regalías de las compañías productoras de alimentos y productos farmacéuti$\cos$, las cuales usan el germoplasma y las plantas medicinales de los campesinos e indígenas.

Está claro que los pueblos indígenas y su diversidad son vistos como materia prima por las corporaciones multinacionales, las cuales han obtenido miles de millones de dólares en semillas desarrolladas en los laboratorios de EE.UU. a partir de germoplasma que los agricultores del tercer mundo mejoraron cuidadosamente por generaciones(9). Por el momento, los campesinos no son recompensados por su milenario conocimiento, mientras las corporaciones multinacionales empiezan a obtener regalías de los países del tercer mundo estimadas en miles de millones de dólares. Hasta ahora las compañías de biotecnología no han recompensado a los agricultores del tercer mundo por la expropiación de sus semillas y recursos genéticos.

Mito 4: La biotecnología conducirá a la conservación de la biodiversidad.

Aunque la biotecnología tiene la capacidad de crear nuevas variedades de plantas comerciales y de esta manera contribuir a la biodiversidad, es difícil que esto suceda. La estrategia de las corporaciones multinacionales consiste en crear amplios mercados internacionales para la semilla de un solo producto. La tendencia es formar mercados internacionales de semillas uniformes. Aún más, las medidas dictadas por las corporaciones multinacionales sobre el sistema de patente que prohíbe a los agricultores rehusar la semilla que rinden sus cosechas, afectará las posibilidades de la conservación in situ y el mejoramiento de la diver- 
sidad genética a nivel local. Los sistemas agrícolas desarrollados con cultivos transgénicos favorecerán los monocultivos que se caracterizan por niveles peligrosos de homogeneidad genética, los cuales conducen a una mayor vulnerabilidad de los sistemas agrícolas al estrés biótico y abiótico(11). Conforme la nueva semilla producida por bioingeniería reemplace a las antiguas variedades tradicionales y a sus parientes silvestres, se acelerará la erosión genética(9). De este modo, la presión por la uniformidad no sólo destruirá la diversidad de los recursos genéticos, sino que también romperá la complejidad biológica que condiciona la sustentabilidad de los sistemas agrícolas tradicionales(12).

Los recientes hallazgos de contaminación de variedades locales de maíz con transgénicos en Oaxaca, México, plantean un dilema de inmensas proporciones ecológicas para los campesinos. El problema es que la dispersión vía flujo genético de características transgénicas a variedades locales, puede diluir la sustentabilidad natural típica de estas variedades criollas. Al expresar la característica transgénica, las variedades criollas lo pueden hacer a expensas de características claves para subsistir en ambientes marginales, tales como resistencias a sequías, heladas o plagas.

Mito 5: La biotecnología no es ecológicamente dañina y dará origen a una agricultura sustentable libre de químicos.

La biotecnología se está desarrollando para "resolver" los problemas causados por las anteriores tecnologías con agroquímicos (resistencia a los plaguicidas, contaminación, degradación del suelo, etc.), los cuales fueron promovidos por las mismas compañías que ahora son líderes de la biorrevolución. Los cultivos transgénicos desarrollados para el control de plagas siguen fielmente el paradigma de los plaguicidas: usar un solo mecanismo de con- trol que ha fallado una y otra vez con insectos, patógenos y malezas(13). Los cultivos transgénicos tienden a incrementar el uso de los plaguicidas y a acelerar la evolución de "super malezas" y plagas de razas de insectos resistentes(14). El enfoque "un gen resistente-una plaga" ha sido superado fácilmente por las plagas, las cuales se adaptan continuamente a nuevas situaciones y sus mecanismos de destoxificación evolucionan(11). Hay muchas preguntas ecológicas sin respuesta respecto al impacto de la liberación de plantas y microorganismos transgénicos en el medio ambiente. Entre los principales riesgos asociados con las plantas obtenidas por ingeniería genética está la transferencia no intencional de los "transgenes" a parientes silvestres de los cultivos y los efectos ecológicos impredecibles que esto implica(14). Además, se sabe que las toxinas del maíz Bt, por ejemplo, impactan a otros insectos benéficos que ejercen control biológico sobre plagas y que, además, se acumulan en el suelo afectando la fauna edáfica que descompone la materia orgánica y regula el reciclaje de nutrientes. La eliminación de esta biodiversidad puede ser de alta significación para los campesinos que dependen de los servicios ecológicos de la biodiversidad.

Por las consideraciones mencionadas, la teoría agroecológica predice que la biotecnología exacerbará los problemas de la agricultura convencional $y$, al promover los monocultivos, también socavará los métodos ecológicos de manejo agrícola, tales como la rotación y los policultivos(1). Como está concebida en la actualidad, la biotecnología no se adapta a los amplios ideales de una agricultura sustentable.

Mito 6: La biotecnología mejorará el uso de la biología molecular para beneficio de todos los sectores de la sociedad.

La demanda por la nueva biotecnología no surgió como un resultado de demandas socia- 
les, sino de cambios en las leyes de patentes y de los intereses de lucro de las compañías de químicos para enlazar semillas y plaguicidas. La tecnología surgió a partir de los sensacionales avances de la biología molecular y de la disponibilidad de capitales aventureros para arriesgar, como resultado de leyes de impuestos favorables. El peligro está en que el sector privado está influyendo en la dirección de la investigación del sector público en una forma sin precedentes. En la medida en que más universidades e institutos públicos de investigación se asocian con las corporaciones, aparecen cuestiones éticas más serias sobre quién es dueño de los resultados de la investigación y qué investigaciones se realizan. La tendencia de los investigadores universitarios involucrados en tales asociaciones a guardar el secreto, trae a colación preguntas sobre ética personal y sobre conflictos de intereses.

En muchas universidades, la habilidad de un profesor para atraer la inversión privada es, a menudo, más importante que las calificaciones académicas, eliminando los incentivos para que los científicos sean responsables ante la sociedad. Las áreas que no atraen el apoyo corporativo, como el control biológico y la agroecología, están siendo dejadas de lado y esto no favorece el interés público. Lo trágico es que, dados los regímenes existentes de propiedad intelectual controlados por corporaciones, las instituciones públicas no pueden liberar cultivos transgénicos que ellos desarrollen porque la mayoría de las tecnologías adyuvantes (vectores, marcadores, etc.) estan patentadas. Para liberar un producto las instituciones tendrían que pagar millones de dólares por "royalties" a corporaciones que poseen las varias patentes.

\section{Agroecología: alternativa real}

Los defensores de la Revolución Verde sostienen que los países de América Latina deberían optar por un modelo industrial basado en variedades mejoradas y en el creciente uso de fertilizantes y pesticidas, a fin de proporcionar una provisión adicional de alimentos a sus crecientes poblaciones y economías. El problema es que la biotecnología no reduce el uso de agroquímicos ni aumenta los rendimientos. Tampoco beneficia a los consumidores ni a los agricultores pobres. Dado este escenario, un creciente número de agricultores, ONGs y otros propulsores de la agricultura sostenible propone que, en lugar de este enfoque intensivo en capital e insumos, los países de la región deberían propiciar un modelo agroecológico que da énfasis a la biodiversidad, el reciclaje de los nutrientes, la sinergia entre cultivos, animales, suelos y otros componentes biológicos, así como a la regeneración y conservación de los recursos.

Una estrategia de desarrollo agrícola sostenible que mejora el medio ambiente debe estar basada en principios agroecológicos y en un método participativo en el desarrollo y difusión de tecnología. La agroecología es la ciencia que se basa en los principios ecológicos para el diseño y manejo de sistemas agrícolas sostenibles y de conservación de recursos, y que ofrece muchas ventajas para el desarrollo de tecnologías más favorables para el agricultor. La agroecología se erige sobre el conocimiento indígena y tecnologías modernas selectas de bajos insumos para diversificar la producción. El sistema incorpora principios biológicos y los recursos locales para el manejo de los sistemas agrícolas, proporcionando a los pequeños agricultores una forma ambientalmente sólida y rentable de intensificar la producción en áreas marginales.

Se estima que, a nivel global, aproximadamente 1,9 a 2,2 mil millones de personas aún no han sido tocadas directa o indirectamente por la tecnología agrícola moderna. En América Latina la proyección es que la población rural permanecería estable en 125 millones hasta el año 2000, pero 
el $61 \%$ de esta población es pobre y la expectativa es que aumente. La mayor parte de la pobreza rural (cerca de 370 millones) se centra en áreas de escasos recursos, muy heterogéneas y predispuestas a riesgos. Sus sistemas agrícolas son de pequeña escala, complejos y diversos. La mayor pobreza se encuentra con más frecuencia en las zonas áridas o semiáridas, y en las montañas y laderas que son vulnerables desde el punto de vista ecológico. Tales fincas y sus complejos sistemas agrícolas constituyen grandes retos para los investigadores.

Para que beneficie a los campesinos pobres, la investigación y el desarrollo agrícolas deberían operar sobre la base de un enfoque "de abajo hacia arriba", usando y construyendo sobre los recursos disponibles -la población local, sus conocimientos y sus recursos naturales nativos. Deben tomarse muy en serio las necesidades, aspiraciones y circunstancias particulares de los pequeños agricultores, por medio de métodos participativos. Esto significa que desde la perspectiva de los agricultores pobres, las innovaciones tecnológicas deben:

- Ahorrar insumos y reducir costos

- Reducir riesgos

- Expandirse hacia las tierras marginales frágiles

- Ser congruentes con los sistemas agrícolas campesinos

- Mejorar la nutrición, la salud y el medio ambiente

Precisamente, debido a estos requerimientos la agroecología ofrece más ventajas que la Revolución Verde y los métodos biotecnológicos. Las características promisorias de las técnicas agroecológicas son que:

- Se basan en el conocimiento indígena y la racionalidad campesina

- Son económicamente viables, accesibles y basadas en recursos locales
- Son sanas para el medio ambiente y sensibles desde el punto de vista social y cultural

- Evitan el riesgo y se adaptan a las condiciones del agricultor

- Mejoran la estabilidad y la productividad total de la finca y no sólo de cultivos particulares

Hay miles de casos de productores rurales que, en asociación con ONGs y otras organizaciones, promueven sistemas agrícolas y conservan los recursos manteniendo altos rendimientos y cumpliendo con los criterios antes mencionados. Aumentos de 50 a $100 \%$ en la producción son bastante comunes con la mayoría de estos métodos de producción. En ocasiones, los rendimientos de los cultivos que constituyen el sustento de los pobres -arroz, frijoles, maíz, yuca, papa, cebada- se han multiplicado gracias al trabajo y al conocimiento local más que a la compra de insumos costosos, y capitalizando sobre los procesos de intensificación y sinergia. Más importante tal vez que sólo los rendimientos, es posible aumentar la producción total en forma significativa diversificando los sistemas agrícolas, usando al máximo los recursos disponibles.

Muchos ejemplos sustentan la efectividad de la aplicación de la agroecología en el mundo en desarrollo. Se estima que alrededor de 1,45 millones de familias rurales pobres que viven en 3,25 millones de hectáreas han adoptado tecnologías regeneradoras de los recursos, incluyendo en Brasil 200000 agricultores que usan abonos verdes y cultivos de cobertura duplicando el rendimiento del maíz y el trigo, y en Guatemala-Honduras donde 45000 agricultores usaron la leguminosa Mucuna como cobertura para la conservación del suelo, triplicando los rendimientos del maíz en las laderas. En México, aproximadamente 100000 pequeños productores de café orgánico aumentaron su producción en un $50 \%$. 


\section{Conclusiones}

A fines de la década de los años 80, una publicación de Monsanto indicaba que, en el futuro, la biotecnología revolucionaría a la agricultura con productos basados en los métodos propios de la naturaleza, haciendo que el sistema agrícola fuera más amigable para el medio ambiente y más provechoso para el agricultor(15). Más aún, se proporcionarían plantas con defensas genéticas autoincorporadas contra insectos y patógenos. Desde entonces, muchos otros han prometido varias otras recompensas que la biotecnología puede brindar a través del mejoramiento de cultivos. El problema ético reside en que muchas de estas promesas son infundadas y muchas de las ventajas o beneficios de la biotecnología no han podido ser o no han sido hechos realidad.

Aunque es claro que la biotecnología puede ayudar a mejorar la agricultura, dada su actual orientación la biotecnología promete más bien daños al medio ambiente, una mayor industrialización de la agricultura y una intrusión más profunda de intereses privados en la investigación del sector público. Hasta ahora la dominación económica y política de las corporaciones multinacionales en la agenda del desarrollo agrícola ha tenido éxito a expensas de los intereses de los consumidores, los campesinos, las pequeñas fincas familiares, la vida silvestre y el medio ambiente.

Para la sociedad civil es urgente tener una mayor participación en las decisiones tecnológicas, para que el dominio que ejercen los inte- reses corporativos sobre la investigación científica sea balanceado por un control público más estricto. Las organizaciones públicas, nacionales e internacionales, tales como FAO, CGIAR, etc., tendrán que monitorear y controlar que los conocimientos aplicados no sean propiedad del sector privado, para proteger que tal conocimiento continúe en el dominio público y en beneficio de las sociedades rurales. Deben desarrollarse regímenes de regulación controlados públicamente y emplearlos para monitorear y evaluar los riesgos sociales y ambientales de los productos de la biotecnología.

Finalmente, la tendencia hacia una visión reduccionista de la naturaleza y de la agricultura, promovida por la biotecnología contemporánea, debe ser revertida por un enfoque más holístico, para asegurar que las alternativas agroecológicas no sean ignoradas y que sólo se investiguen y desarrollen aspectos biotecnológicos ecológicamente aceptables. Ha llegado el momento de enfrentar efectivamente el reto $\mathrm{y}$ la realidad de la ingeniería genética. Como ha ocurrido con los plaguicidas, las compañías de biotecnología deben sentir el impacto de los movimientos ambientalistas, laborales y campesinos, de modo que reorienten su trabajo para beneficio de toda la sociedad y la naturaleza. El futuro de la investigación con base en la biotecnología estará determinado por relaciones de poder y no hay razón para que los agricultores y el público en general, si se organizan y logran suficiente poder, no puedan influir en la dirección de la biotecnología, de modo que cumpla con las metas de la sustentabilidad. 


\section{Referencias}

1. Hindmarsh R. The flawed "sustainable" promise of genetic engineering. The Ecologist 1991; 21: 196205.

2. Busch L, Lacy WB, Burkhardt J, et al. Plants, Power and Profit. Oxford: Basil Blackwell; 1990.

3. Krimsky S, Wrubel RP. Agricultural Biotechnology and the Environment: Science, Policy and Social Issues. Urbana: University of Illinois Press; 1996.

4. Lappé FM, Collins J, Rosset P. World hunger: twelve myths. New York: Grove Press; 1998.

5. Aristide JB. Eyes of the heart: seeking a path for the poor in the age of globalisation. Monroe ME: Common Courage Press; 2000.

6. Altieri MA, Rosset P, Thrupp LA. The potential of agroecology to combat hunger in the developing world. (2020 Brief $\mathrm{N}^{\circ}$ 55). Washington DC: International Food Policy Research Institute; 1998.

7. Uphoff N, Altieri MA. Alternatives to conventional modern agriculture for meeting world food needs in the next century. Report of a Bellagio Conference. Ithaca, NY: Cornell International Institute for Food, Agriculture and Development; 1999.

8. Rosset P. The Multiple Functions And Benefits Of Small Farm Agriculture In The Context Of Global Trade Negotiations (Food First Policy Brief $\mathrm{N}^{\circ} 4$ ). Oakland, CA: Institute for Food and Development Policy; 1999.

9. Fowler C, Mooney P. Shattering: Food, Politics and the Loss of Genetic Diversity. Tucson: University of Arizona Press; 1990.

10. Greenland DJ. The sustainability of rice farming. Wallingford, UK: CAB International; 1997.

11. Robinson RA. Return to Resistance: Breeding Crops to Reduce Pesticide Resistance. Davis: AgAccess; 1996.

12. Altieri MA. Biodiversity and pest management in agroecosystems. New York: Haworth Press; 1994.

13. National Research Council. Ecologically Based Pest Management. Washington DC: National Academy of Sciences; 1996.

14. Rissler J, Mellon M. The Ecological Risks of Engineered Crops. Cambridge: MIT Press; 1996.

15. Office of Technology Assessment. A New Technological Era for American Agriculture. Washington DC: USA Government Printing Office; 1992. 


\section{Bibliografía}

Altieri MA. Agroecology: the science of sustainable agriculture. Boulder: Westview Press; 1996.

Altieri MA. The ecological impacts of transgenic crops on agroecosystem health. Ecosystem Health 2000; 6:13-23.

Altieri MA. Developing sustainable agricultural systems for small farmers in Latin America. Natural Resources Forum 2000; 24: 97-105.

Conway GR. The doubly green revolution: food for all in the twenty-first century. London: Penguin Books; 1997.

Darmancy H. The impact of hybrids between genetically modified crop plants and their related species: introgression and weediness. Molecular Ecology 1994; 3: 37-40.

Donnegan KK, Seidler RJ. Effects of transgenic plants on soil and plant micro-organisms. Recent Research Developments in Microbiology 1999; 3: 415-424.

Goldberg RJ. Environmental concerns with the development of herbicide-tolerant plants. Weed Technology 1992; 6: 647-652.

Gould F. Potential and problems with high-dose strategies for pesticidal engineered crops. Biocontrol Science and Technology 1994; 4: 451-461.

Hilbeck A, Moar WJ, Putzai-Carey M, et al. Prey-mediated effects of Cry1Ab toxin and protoxin on the predator Chrysoperla carnea. Entomologia Experimentalis et Applicata 1999; 91: 305-316.

Holt JS, Powles SB, Holtum JAM. Mechanisms and agronomic aspects of herbicide resistance. Annual Review Plant Physiology Plant Molecular Biology 1993; 44: 203-229.

Jordan CF. Genetic engineering, the farm crisis and world hunger. BioScience 2002; 52: 523-529.

Lappé FM, Bailey B. Against The Grain: Biotechnology And The Corporate Takeover Of Food. Monroe, Maine: Common Courage Press; 1998.

Losey JJE, Rayor LS, Carter ME. Transgenic pollen harms Monarch larvae. Nature 1999; 399: 214.

Lutman PJW, ed. Gene flow and agriculture: relevance for transgenic crops. Staffordshire: British Crop Protection Council Symposium Proceedings N 72; 1999: 43-64.

Obrycki JJ, Losey JE, Taylor OR, et al. Transgenic insecticidal corn: beyond insecticidal toxicity to ecological complexity. BioScience 2001; 353-361.

Palm CJ, Schaller DL, Donegan KK, et al. Persistence in soil of transgenic plant produced Bacillus thuringiensis var. Kustaki d-endotoxin. Canadian Journal of Microbiology 1996; 42: 1258-1262.

Paoletti MG, Pimentel D. Genetic engineering in agriculture and the environment: assessing risks and benefits. BioScience 1996; 46: 665-671.

Persley GJ, Lantin MM, eds. Agricultural biotechnology and the poor. Washington DC: Consultative Group on International Agricultural Research; 2000.

Pretty J. Regenerating agriculture: policies and practices for sustainability and self-reliance. London: Earthscan; 1995. 
Pretty J, Hine R. Feeding the world with sustainable agriculture: a summary of new evidence. Final report from SAFE-World Research Project. Colchester, UK: University of Essex; 2000.

Saxena D, Flores S, Stotzky G. Insecticidal toxin in root exudates from Bt corn. Nature 1999; 40: 480.

Snow AA, Moran P. Commercialization of transgenic plants: potential ecological risks. BioScience 1997; 47: 86-96.

Thrupp LA. Cultivating biodiversity: agrobiodiversity for food security. Washington DC: World Resources Institute; 1998.

USDA. Genetically Engineered Crops for Pest Management. Washington DC: United States Department of Agriculture Economic Research Service; 1999. 